\title{
Deter mination of the Structure of Vitrified Hydroceramic/CBC Waste Form Glasses Manufactured from DOE Reprocessing Waste
}

\author{
Final Report \\ For the Period June 1, 2001 to May 31, 2004 \\ Plus No-Cost Extension Period to May 31, 2005 \\ Grant No. DE-FG07-011D14105 \\ U.S. Department of Energy
}

The Pennsylvania State University

Department of Mechanical and Nuclear Engineering and Materials Research Institute

Barry E. Scheetz and William B. White, Principal Investigators

Marcia Chesleigh, Graduate Student Researcher Alison Portanova Graduate Student Researcher Johnson Olanrewaju, Graduate Student Researcher

May 31, 2005 


\section{BACKGROUND TO THE TECHNICAL PROBLEM}

The selection of a glass-making option for the solidification of nuclear waste has dominated DOE waste form programs since the early 1980's. Both West Valley and Savannah River are routinely manufacturing glass logs from the high level waste inventory in tank sludges. However, for some wastes, direct conversion to glass is clearly not the optimum strategy for immobilization. INEEL, for example, has approximately $4400 \mathrm{~m}^{3}$ of calcined high level waste with an activity that produces approximately 45 watts $/ \mathrm{m}^{3}$, a rather low concentration of radioactive constituents. For these wastes, there is value in seeking alternatives to glass.

An alternative approach has been developed and the efficacy of the process demonstrated that offers a significant savings in both human health and safety exposures and also a lower cost relative to the vitrification option. The alternative approach utilizes the intrinsic chemical reactivity of the highly alkaline waste with the addition of aluminosilicate admixtures in the appropriate proportions to form zeolites. The process is one in which a chemically bonded ceramic is produced. The driving force for reaction is derived from the chemical system itself at very modest temperatures and yet forms predominantly crystalline phases. Because the chemically bonded ceramic requires an aqueous medium to serve as a vehicle for the chemical reaction, the proposed zeolitecontaining waste form can more adequately be described as a hydroceramic. The hydrated crystalline materials are then subject to hot isostatic pressing (HIP) which partially melts the material to form a glass ceramic.

The scientific advantages of the hydroceramic/CBC approach are:

i) Low temperature processing

ii) High waste loading and thus only modest volumetric bulking from the addition of admixtures

iii) Ability to immobilize sodium.

iv) Ability to handle low levels of nitrate $\left(2-3 \% \mathrm{NO}_{3}{ }^{-}\right)$.

v) The flexibility of a vitrifiable waste.

vi) A process that is based on an industry with decades of practical experience.

The research undertaken in the present investigation builds on a previous study under the NEER program. The earlier studies identified an optimal formulation for the immobilization of the calcine that is both compositionally adequate to retain radionuclides as well as hazardous constituents and which has a reaction rate that will allow the technical employment of the process. The study established in a general way 
the glass-forming region in the system $\mathrm{M}_{2} \mathrm{O}-\mathrm{MO}-\mathrm{Al}_{2} \mathrm{O}_{3}-\mathrm{SiO}_{2}\left(\mathrm{M}_{2} \mathrm{O}=\right.$ alkali metal oxides; $\mathrm{MO}=$ alkaline earth metal oxide) which provides the base for these hydroceramic/CBC materials (Fig. 1).

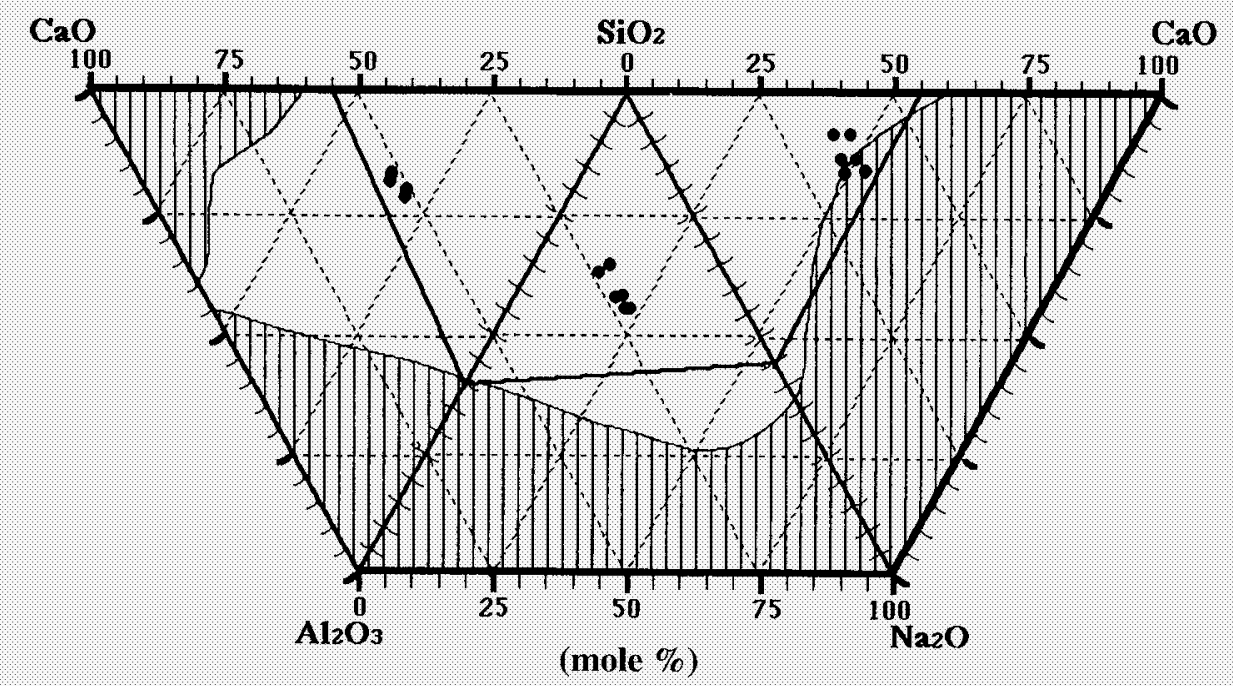

Figure 1. Quaternary composition diagram for the $\mathrm{Al}_{2} \mathrm{O}_{3}-\mathrm{SiO}_{2}-\mathrm{CaO}-\mathrm{Na}_{2} \mathrm{O}$ system including the reported glass-forming composition region limits. The region delineated by the straight lines represents the laboratory developed "rule-of-thumb" limits reported by Gougar et al. (1995).

The objectives of the present program are to track the structural changes that take place during formulation, chemical reaction, and HIPing. Compositions must be varied through the glass-forming region, structures of the crystals and glass matrix of the glassceramic determined, and the structural characteristics in turn related to stability and leachability of the final products.

\section{GLASS SYNTHESIS}

The fabrication of the glasses was accomplished in three basic steps. The first step was to measure and mix the various components. Second, the powder was compacted, usually by sintering, into a form that was strong enough to withstand both being grasped by tongs and the air currents created by the hydrogen torch. Finally, the compacts were melted with a hydrogen torch to create clear glass beads.

The sample glasses are in the $\mathrm{SiO}_{2}-\mathrm{Al}_{2} \mathrm{O}_{3}-\mathrm{Na}_{2} \mathrm{O}-\mathrm{CaO}$ system. To produce the specific glasses, appropriate proportions of $\mathrm{SiO}_{2}, \mathrm{Al}_{2} \mathrm{O}_{3}, \mathrm{Na}_{2} \mathrm{CO}_{3}$, and $\mathrm{CaCO}_{3}$ were weighed out. The mass of each of these starting materials necessary to create the desired glass was calculated using an excel spreadsheet. The actual powder masses were weighed to within 0.00003 grams of the calculated values. The components were then mixed using a mortar and pestle. 
Two methods were used to create a sturdy sample, depending on whether the composition would sinter in the furnace (maximum temperature of $866^{\circ} \mathrm{C}$ ). For the compositions that do sinter, a trough was carved out of a silica brick and a piece of platinum foil placed into this trough, creating a " $U$ " shape. The powder mixture was placed into the trough of the foil, and then placed into the furnace for approximately 24 hours. After sintering had taken place, the samples were removed from the furnace and allowed to cool. The platinum foil was removed from the sintered rod. For the compositions that do not sinter in the $866^{\circ} \mathrm{C}$ furnace, the powder was placed into a pellet mold and hydraulically pressed into pellets using pressures of approximately $6 \mathrm{kpsi}$. These pellets were placed onto a piece of platinum foil, and then put into the furnace for approximately 24 hours.

After the samples (either the sintered rod, or the pellet) were removed from the furnace, they were melted using a hydrogen torch. During the melting process, tiny glass beads were created, and would eventually, with the force of the air from the torch, detach from the bulk of the sample.

Table 1 shows the compositions that have been created by the process described above. An asterisk designates the compositions that were created without the use of the pellet press. The compositions are shown as atomic percent of the final glass. The compositions are displayed graphically in Figure 2. To plot quaternary compositions on a triangular diagram, the alkali, $\mathrm{Na}_{2} \mathrm{O}$, and alkaline earth, $\mathrm{CaO}$, components were added and plotted together. Thus each point on Figure 2 may represent a number of glasses varying in their $\mathrm{Na}_{2} \mathrm{O} / \mathrm{CaO}$ ratio.

\section{RAMAN SPECTROSCOPY}

\section{A. Spectroscopic Methods}

The Raman Spectrometer used for this research was generously made available by the Department of Physics and Astronomy at the Arizona State University. The system is a SPEX 1877 Triplemate spectrometer. The spectrometer utilizes an argon laser with a wavelength of $514.5 \mathrm{~nm}$. The laser power at the sample is less than $4 \mathrm{~mW}$ with a spot size between 3 and 5 microns. The spectrometer has a microfocus system with an Olympus 20X 0.4 NA objective, and a CCD detector with 200 micron slits. Data collection was performed by a computer running the SPEX 3000 program.

The microscope stage required a flat surface for spectroscopic measurements. Using Crystalbond-509, the glass beads were affixed to a sample-polishing fixture. The beads were polished with silicon carbide 320 sandpaper until the desired flat surface area is achieved. After the first flat surface was achieved, the crystal bond was dissolved in acetone until the bead was clean. The process was repeated to create the second flat surface, ensuring that the first polished surface was flat against the fixture. 
Table 1: Compositions of Investigated Glasses

\begin{tabular}{|r|c|c|c|c|c|}
\hline & & $\mathrm{SiO}_{2}$ & $\mathrm{Na}_{2} \mathrm{O}$ & $\mathrm{Al}_{2} \mathrm{O}_{3}$ & $\mathrm{CaO}$ \\
\hline 1 & & 60 & 0 & 20 & 20 \\
\hline 2 & $\mathrm{~F}$ & 30 & 40 & 30 & 0 \\
\hline 3 & & 70 & 30 & 0 & 0 \\
\hline 4 & $\mathrm{~F}$ & 40 & 30 & 30 & 0 \\
\hline 5 & & 70 & 10 & 0 & 20 \\
\hline 6 & $\mathrm{~F}$ & 40 & 10 & 20 & 30 \\
\hline 7 & $\mathrm{~F}$ & 50 & 30 & 10 & 10 \\
\hline 8 & & 40 & 10 & 40 & 10 \\
\hline 9 & $\mathrm{~F}$ & 40 & 10 & 10 & 40 \\
\hline 10 & $\mathrm{~F}$ & 40 & 40 & 10 & 10 \\
\hline 11 & $\mathrm{~F}$ & 40 & 30 & 10 & 20 \\
\hline 12 & $\mathrm{~F}$ & 40 & 20 & 10 & 30 \\
\hline 13 & & 40 & 30 & 20 & 10 \\
\hline 14 & & 40 & 20 & 30 & 10 \\
\hline 15 & & 40 & 10 & 30 & 20 \\
\hline 16 & & 50 & 20 & 10 & 20 \\
\hline 17 & & 50 & 10 & 20 & 20 \\
\hline 18 & & 50 & 20 & 20 & 10 \\
\hline 19 & & 50 & 10 & 10 & 30 \\
\hline 20 & & 50 & 10 & 30 & 10 \\
\hline 21 & & 60 & 20 & 10 & 10 \\
\hline 22 & & 60 & 10 & 10 & 20 \\
\hline 23 & & 60 & 10 & 20 & 10 \\
\hline 24 & & 70 & 10 & 10 & 10 \\
\hline 25 & $\mathrm{NGF}$ & 30 & 10 & 50 & 10 \\
\hline 26 & $\mathrm{~F}$ & 30 & 20 & 40 & 10 \\
\hline 27 & $\mathrm{~F}$ & 30 & 10 & 40 & 20 \\
\hline 28 & $\mathrm{~F}$ & 30 & 30 & 30 & 10 \\
\hline 29 & $\mathrm{~F}$ & 30 & 20 & 30 & 20 \\
\hline 30 & $\mathrm{~F}$ & 30 & 10 & 30 & 30 \\
\hline 31 & $\mathrm{~F}$ & 30 & 40 & 20 & 10 \\
\hline 32 & $\mathrm{~F}$ & 30 & 30 & 20 & 20 \\
\hline 33 & $\mathrm{~F}$ & 30 & 20 & 20 & 30 \\
\hline 34 & & 30 & 10 & 20 & 40 \\
\hline 35 & $\mathrm{~F}$ & 30 & 50 & 10 & 10 \\
\hline 36 & $\mathrm{~F}$ & 30 & 40 & 10 & 20 \\
\hline 37 & $\mathrm{~F}$ & 30 & 30 & 10 & 30 \\
\hline & & & & & \\
\hline
\end{tabular}

\begin{tabular}{|c|c|c|c|c|c|}
\hline 38 & NGF & 30 & 20 & 10 & 40 \\
\hline 39 & NGF & 30 & 10 & 10 & 50 \\
\hline 40 & NGF & 20 & 10 & 50 & 20 \\
\hline 41 & NGF & 20 & 20 & 50 & 10 \\
\hline 42 & F & 20 & 10 & 40 & 30 \\
\hline 43 & & 20 & 20 & 40 & 20 \\
\hline 44 & & 20 & 30 & 40 & 10 \\
\hline 45 & & 20 & 10 & 30 & 40 \\
\hline 46 & Miss & 20 & 20 & 30 & 30 \\
\hline 47 & & 20 & 30 & 30 & 20 \\
\hline 48 & & 20 & 40 & 30 & 10 \\
\hline 49 & & 20 & 10 & 20 & 50 \\
\hline 50 & & 20 & 20 & 20 & 40 \\
\hline 51 & NGF & 20 & 30 & 20 & 30 \\
\hline 52 & NGF & 20 & 40 & 20 & 20 \\
\hline 53 & NGF & 20 & 50 & 20 & 10 \\
\hline 54 & & 10 & 10 & 30 & 50 \\
\hline 55 & & 10 & 10 & 40 & 40 \\
\hline 56 & F & 10 & 20 & 30 & 40 \\
\hline
\end{tabular}




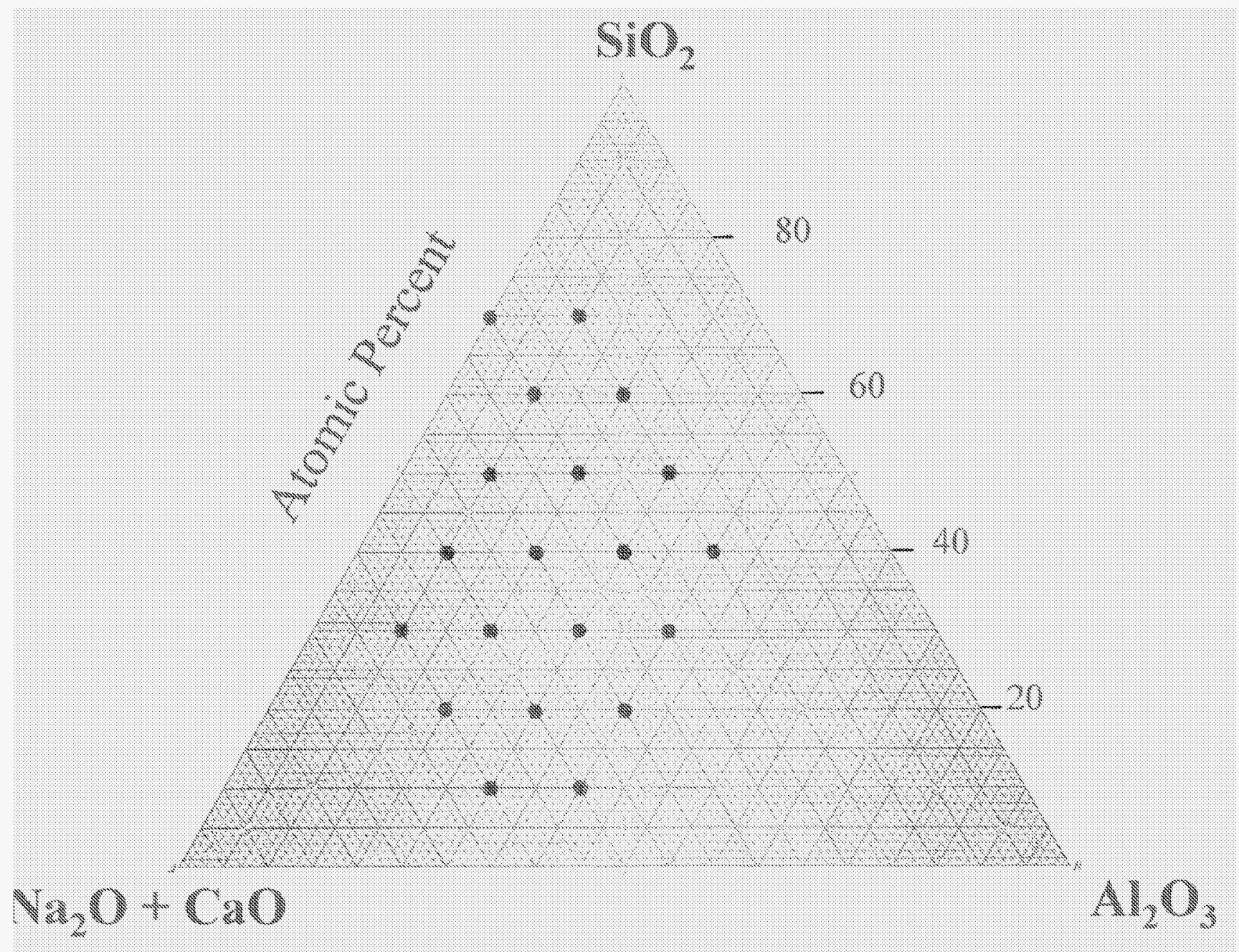

Figure 2. Glass compositions in atomic percent projected onto the $\left(\mathrm{Na}_{2} \mathrm{O}+\mathrm{CaO}\right)-$ $\mathrm{Al}_{2} \mathrm{O}_{3}-\mathrm{SiO}_{2}$ ternary diagram. 
The glass beads were placed on the sample stage, a filter placed into the laser beam to reduce its power, and a mirror adjusted to send the reflected beam directly to the imaging system. This enables the microscope imaging system to be used without overloading its camera. The laser beam was focused onto the flat surface of the bead. For some of the glass samples that fluoresce, the laser was focused just below the surface, to reduce the effects of surface contamination. Once the sample had been brought into focus, the filter and mirror were removed from the beam and the reflected beam from the sample sent directly to the monochromator. The parameters of the scan, such as scan length and range, were then input into the computer for the beginning of the run.

\section{B. Processing the Raman Spectra}

To prepare the final spectra, the data were processed to remove artifacts in the scans. In all of the spectra there exists a sharp drop off of intensity at the high wave number end of the scan beginning at around $1350 \mathrm{~cm}^{-1}$. This drop off is caused by the sensitivity limit of the CCD detector. Furthermore, sharp peaks only a few wavenumbers wide occur in some of the spectra. These peaks are known as cosmic spikes and are an instrument artifact, not part of the glass spectra. These two types artifacts are labeled in Figures 3 and 4, using the spectra for glass sample \#3 and \#17. The points of the drop off and the cosmic peaks were deleted from the Excel spreadsheet to obtain more realistic spectra for further analysis.

The analysis of the scans was accomplished with the Peakfit program, using the "Autofit Peaks I Residuals" option. Hidden peaks, defined as peaks that do not produce local maxima in the data, are found by using the residuals method. This method subtracts the amplitude of the peaks from that of the data stream. Residual peaks are then placed in areas where large amplitudes are left unaccounted for by the main peaks. The peaks were fitted with Gaussian line shapes while allowing the peak width to vary. There is no intrinsic reason that Raman bands should be Gaussian but spectral fitting using Gaussian band shapes has proved useful (Mysen et al., 1982). Peakfit also determined an appropriate baseline to subtract from the data. In Figures 5 and 6, the data are displayed after adjustments have been made for artifacts and the Peakfit determined baseline constructed. With these options selected, Peakfit performed an autoscan, placing peaks in the data stream. With this initial peak estimation, both the smoothing percent and the amplitude threshold were adjusted until the spectra displayed the appropriate line shapes. The smoothing percentage, using the Savitsky-Golay method, determines the smoothing window. The amplitude threshold was adjusted to weed out the small, meaningless peaks that occur, while ensuring that the low amplitude, meaningful peaks remain in the spectra. After adjusting these values, the spectra were modified further by hand. The hand adjustments were performed so the accumulation of the peaks found would better approximate the data. Any peaks that seem to be superfluous in the spectra were deleted. Furthermore, the peaks' amplitude, center and width are adjusted by hand to better approximate the data. Once realistic spectral line shapes were obtained, the Peak Fit algorithm was used to further adjust the peak characteristics to more closely resemble the data. The algorithm uses the least squares minimization, a full curvature matrix 
evaluation, and is set to fit every point of the scan. The Peak Fit algorithm was run and repeated until either an $r^{2}$ value of 0.991 was obtained or until the peaks begin to shift away from the believed value. Figures 7 and 8 show the final spectra determined from this process superimposed onto the data stream after the baseline has been subtracted from it.

A problem encountered in the Raman spectrometry was fluorescence of the glass samples. The fluorescing samples can be identified from the spectra by their large slope of the background line. In Table 2.1, the samples that fluoresce are labeled. These spectra produce significantly less accurate peak values. Figures 9 and 10 show two spectra that exhibit fluorescence, samples \#6 and \#10.

C. Results

Raman spectra were measured for the entire suite of synthesized glasses. Eighteen of these spectra are displayed in Figures $11-14$. These are arranged systematically to show the changes in spectra with composition.

Figure 7 shows the spectrum of a binary alkali silicate glass, $70 \mathrm{SiO}_{2} .30 \mathrm{Na}_{2} \mathrm{O}$, which may be taken as a reference for a highly ordered glass structure. There are two primary features: an intense band near $1100 \mathrm{~cm}^{-1}$ which is assigned to a stretching mode of the $\mathrm{SiO}_{4}$ tetrahedron and an intense band near $500 \mathrm{~cm}^{-1}$ which represents some kind of $\mathrm{SiO}_{4}$ bending mode. This particular composition can be rewritten $\mathrm{Na}_{2} \mathrm{O} .2 .33 \mathrm{SiO}_{2}$ $(\mathrm{NBO} / \mathrm{T}=0.86)$, slightly more polymerized than an ideal sodium disilicate which would be a glass containing, on the average, one non-bridging oxygen per tetrahedron.

The spectra in Figure 11 are of glasses with a fixed alkali and alkaline earth content of 20 mole percent total and a constant $1: 1 \mathrm{Na}_{2} \mathrm{O} / \mathrm{CaO}$ ratio. The silica content decreases from 70 to 40 mole percent while the alumina content increases from 10 to 40 mole percent. The high wavenumber band is greatly broadened from the equivalent band in the alkali silicate spectrum. Ten percent alumina is sufficient to reduce the peak wavenumber from 1066 down to $1010 \mathrm{~cm}^{-1}$. Continued increase in the alumina concentration causes the band to shift down to $983 \mathrm{~cm}^{-1}$ at the highest alumina concentration. The lower wavenumber band takes on a more complex band profile which resolved by the Peak Fit program into at least three components. There also appears a weaker mid-range band near $700 \mathrm{~cm}^{-1}$ which has been correlated with a stretching motion across the oxygens bridging adjacent tetrahedra.

The compositions of the glasses whose spectra are shown in Figure 12 extend to the limits of the glass-forming region. Silica concentrations extend from 50 mole percent to 20 mole percent with alumina varying from 10 to 40 mole percent. The alkali plus alkaline earth content was held at 40 mole percent but it was not possible to keep the $\mathrm{Na}_{2} \mathrm{O} / \mathrm{CaO}$ ratio constant. The high wavenumber band is again very broad but the wavenumber shifts only down to $925 \mathrm{~cm}^{-1}$ at the highest alumina concentration. Again, the bending mode is complex with multiple components. 


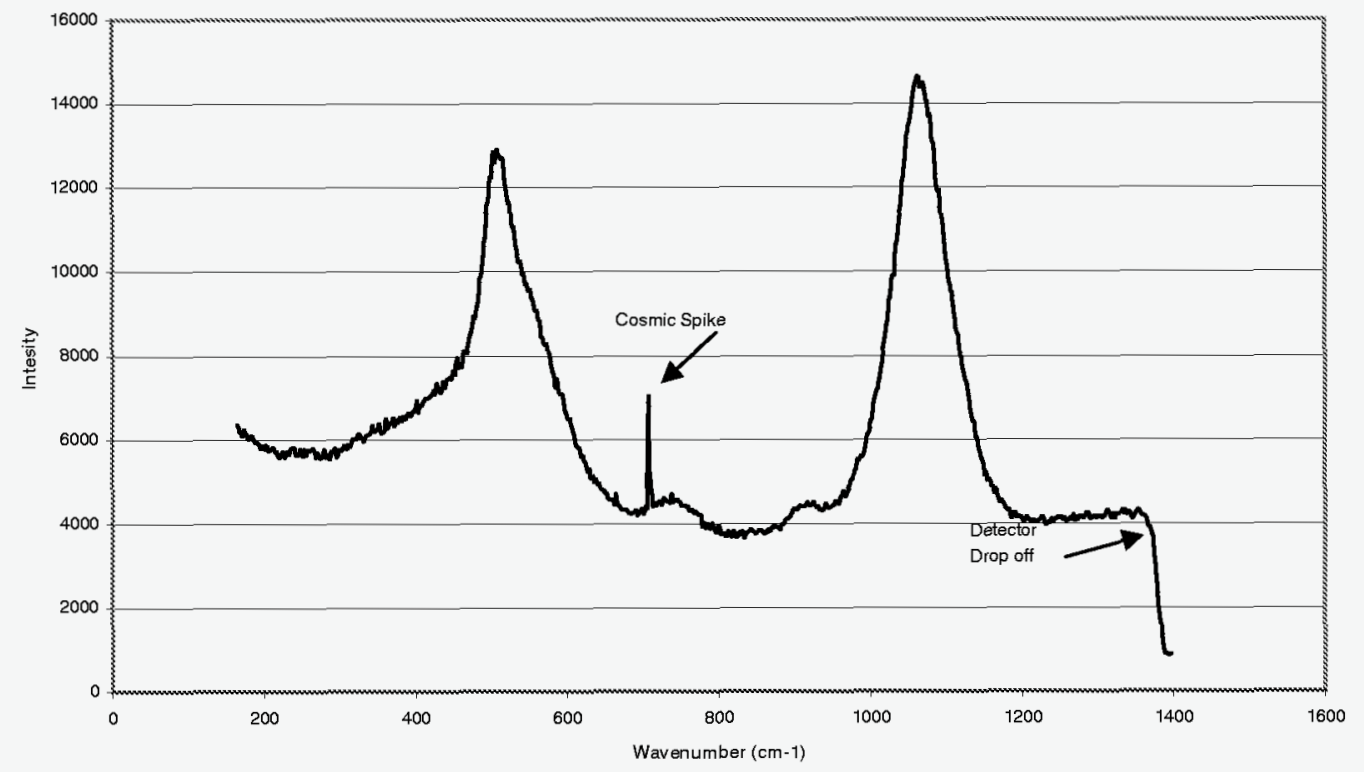

Figure 3. Raw Raman spectrum of glass \#3 showing instrumental artifacts.

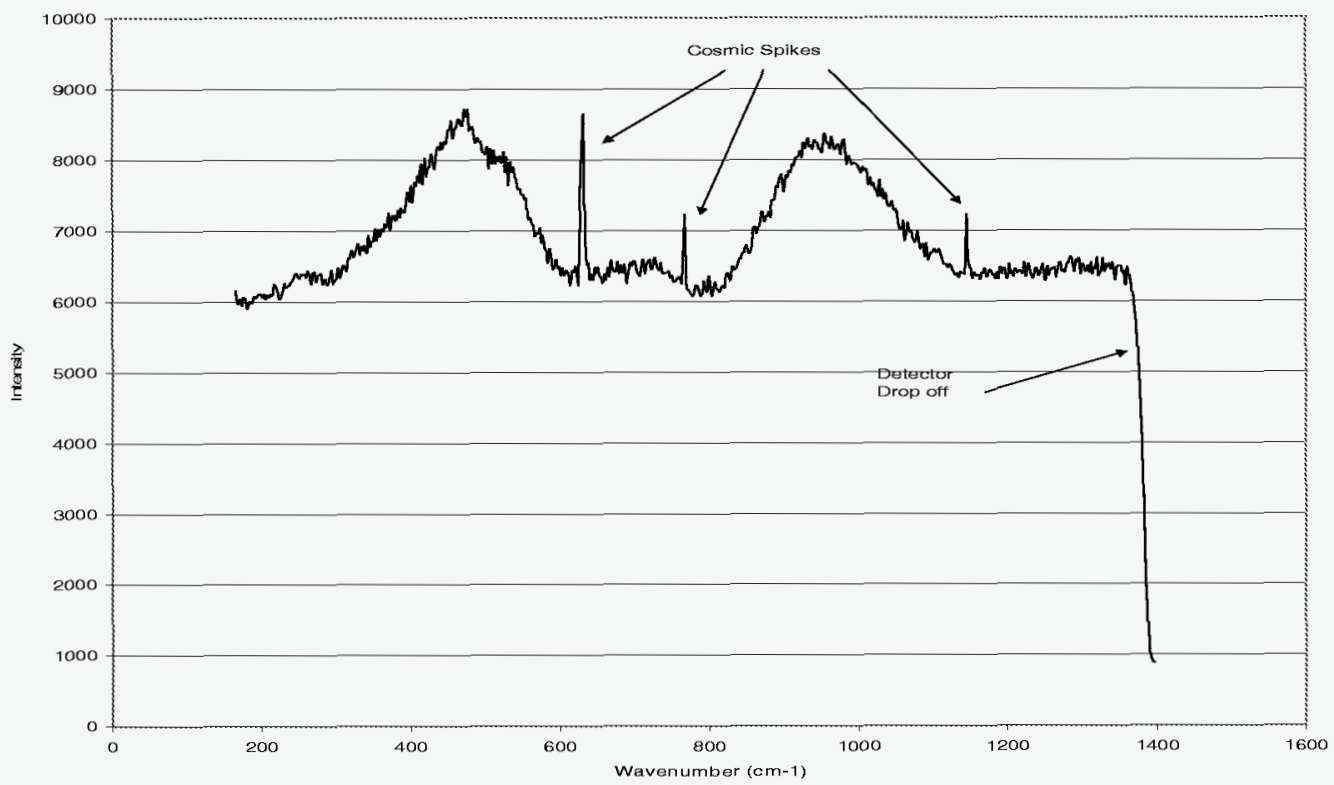

Figure 4. Raw Raman spectrum of glass \#17 showing instrumental artifacts. 


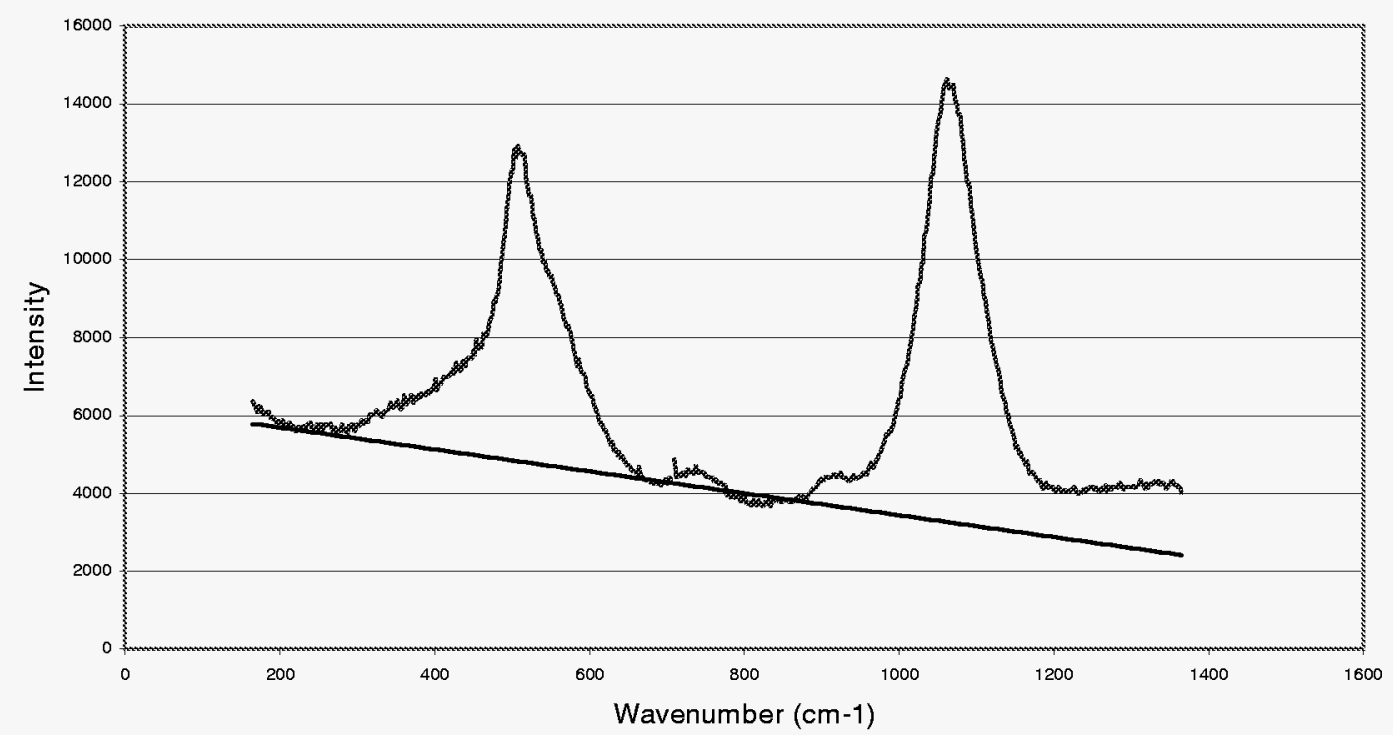

Figure 5. Peak-Fit selected baseline for spectrum of glass \#3.

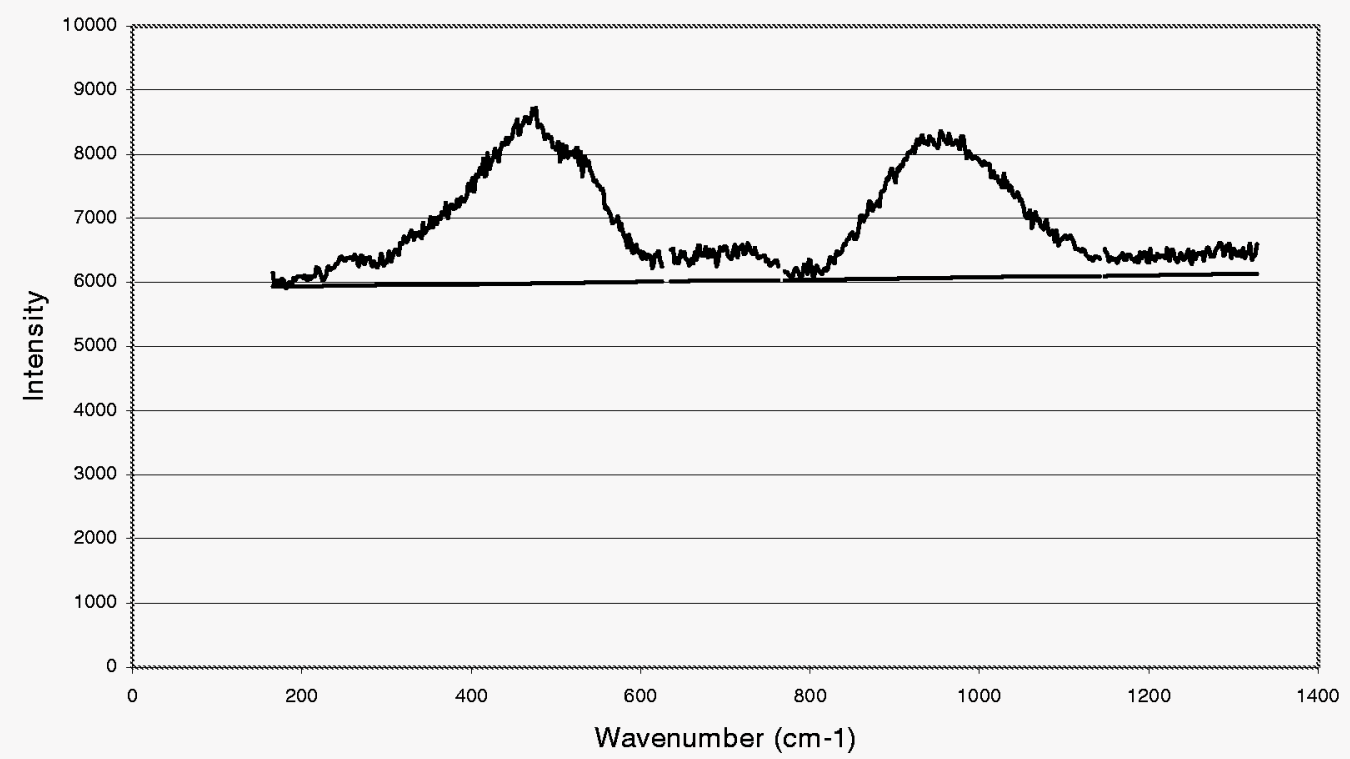

Figure 6. Peak-Fit selected baseline for spectrum of glass \#17. 


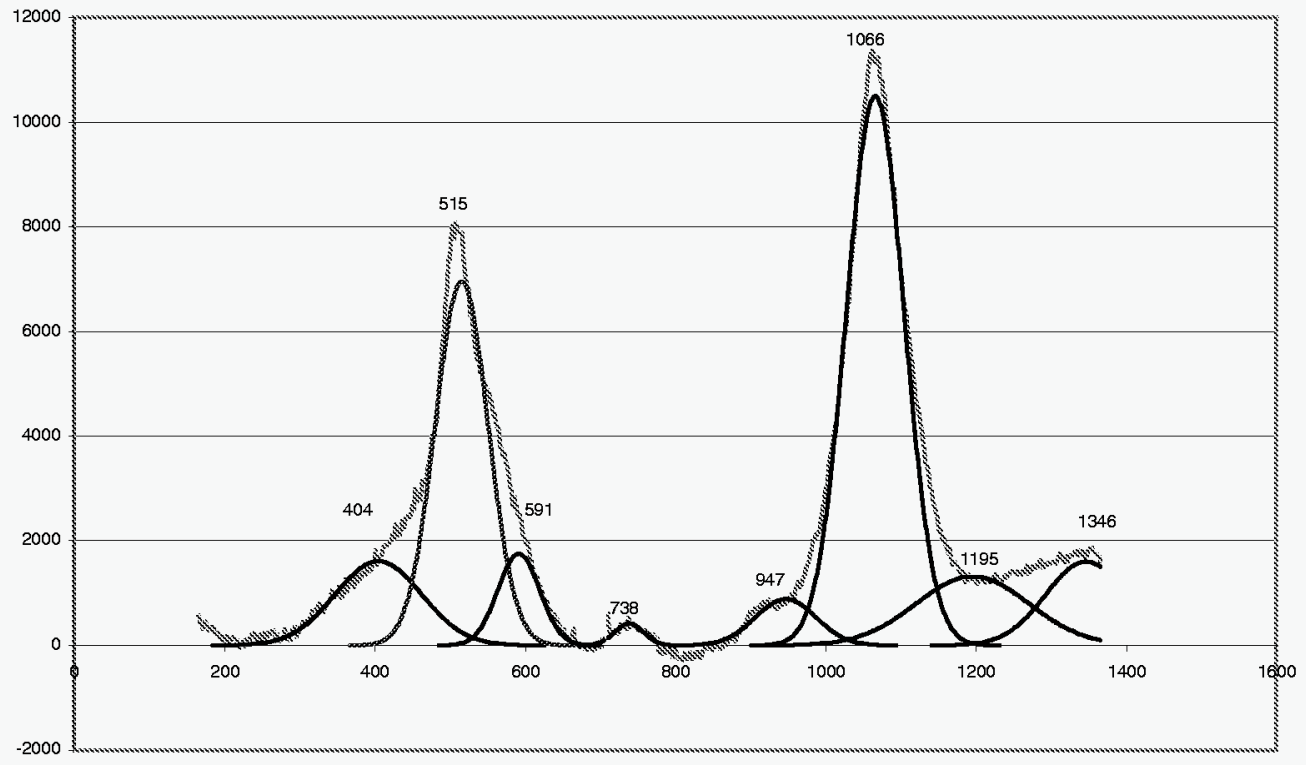

Figure 7. Peak-resolved Raman spectrum for glass \#3.

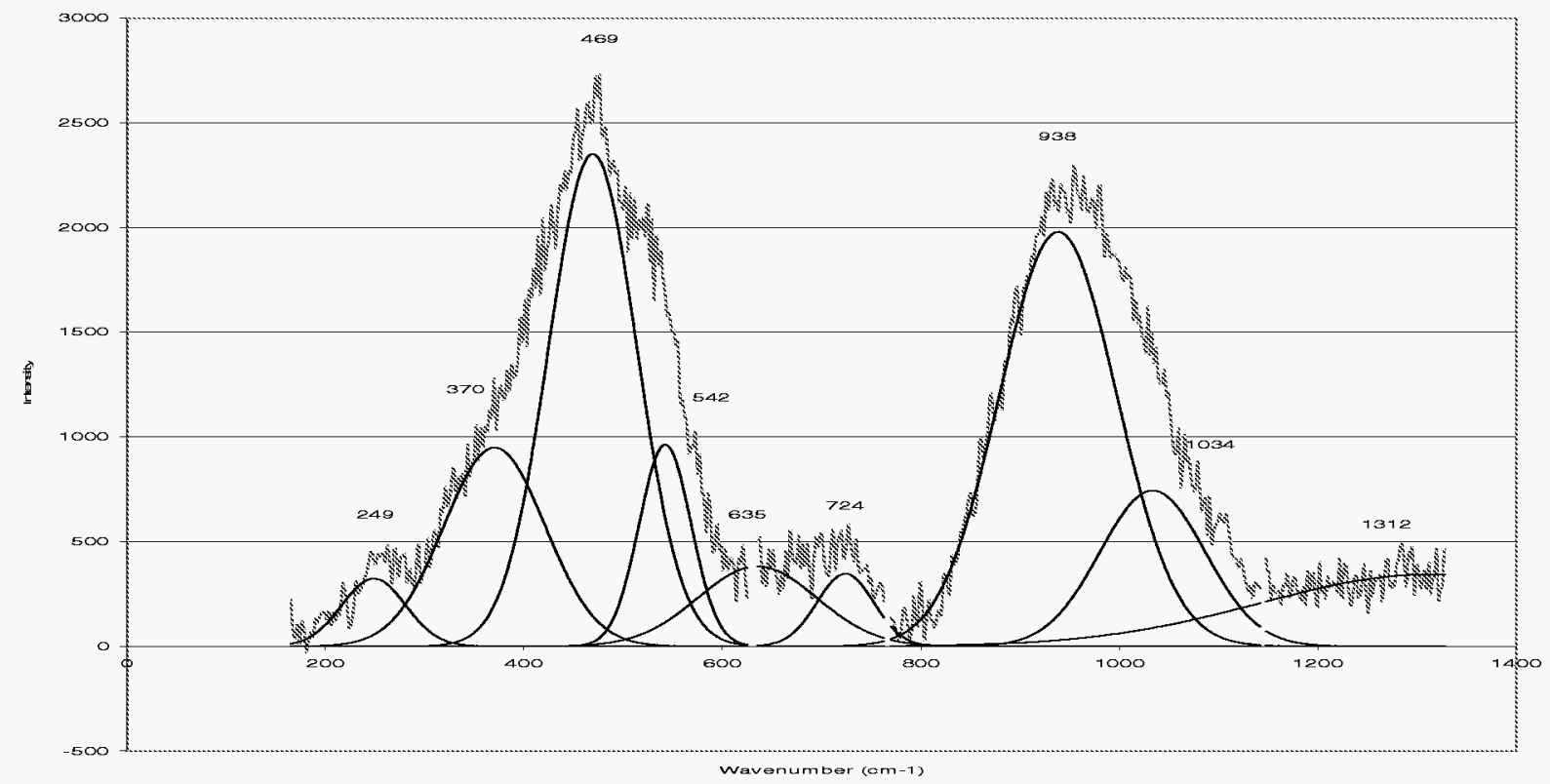

Figure 8. Peak-resolved Raman spectrum for glass \#17. 


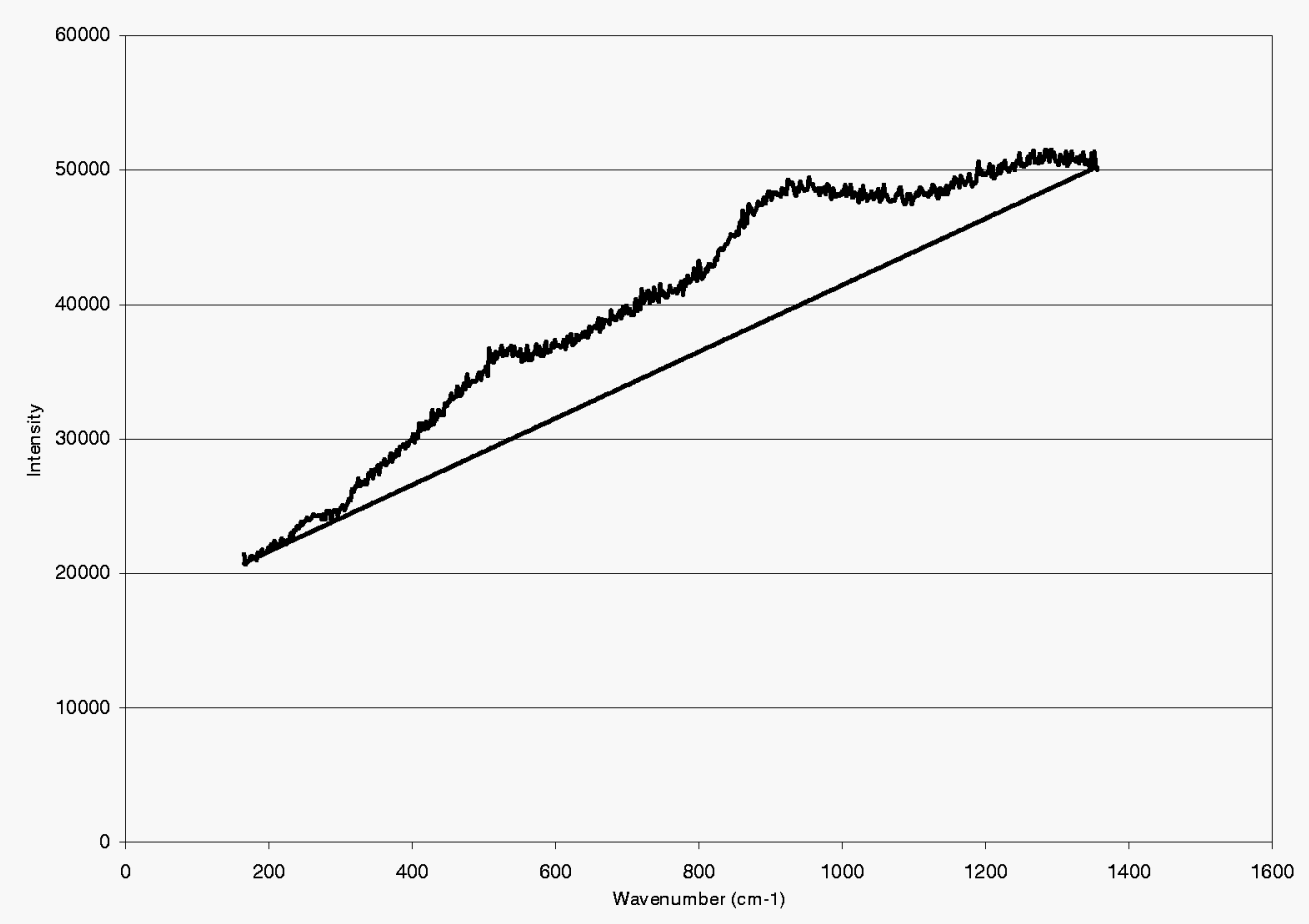

Figure 9. Raw Raman spectrum of glass \#6 showing strong fluorescence background.

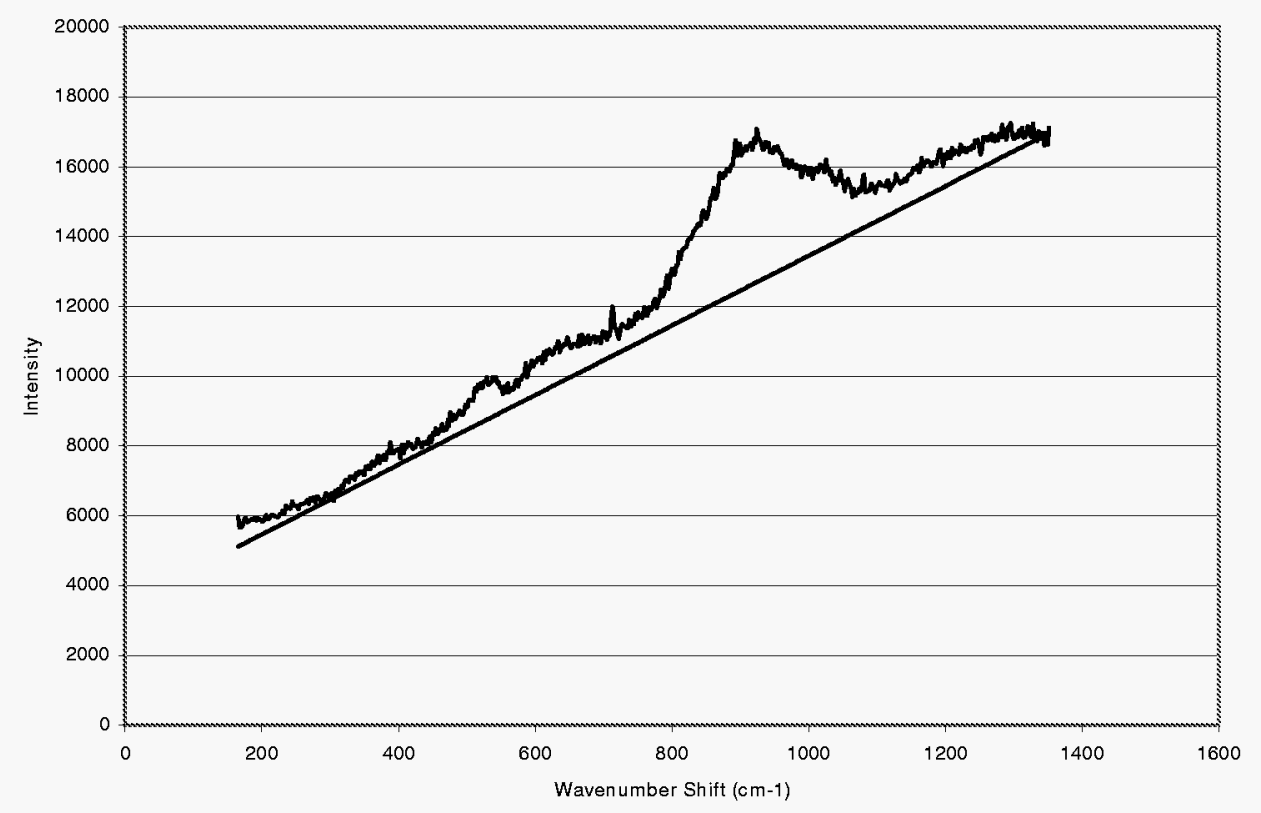

Figure 10. Raw Raman spectrum of glass \#10 showing strong fluorescence background. 
At still higher alkali plus alkaline earth concentrations of 60 mole percent (Fig 13) glasses can be formed with silica content as low as $10 \%$. The range is 30 mole percent $\mathrm{SiO}_{2}$ to 10 mole percent $\mathrm{SiO}_{2}$. The corresponding alumina concentrations vary from 10 to 30 mole percent. The lowest spectrum is Figure 13 is of a glass with $10 \%$ silica and $40 \%$ alumina - essentially an aluminate glass. The spectra of these glasses are rather different from those shown in Figures 11 and 12. The high wavenumber mode is shiftened to the range near $850 \mathrm{~cm}^{-1}$. The high wavenumber band and the low wavenumber band are less distinct and both deconvolute into a number of components.

In the traditional view of glass structure, the role of such large ions as $\mathrm{Na}^{+}$and $\mathrm{Ca}^{2+}$ is to act as network modifiers and to provide charge compensation for $\mathrm{Al}^{3+}$ when it is substituted for $\mathrm{Si}^{4+}$ in the network. Because one mole of $\mathrm{Na}_{2} \mathrm{O}$ provides the same number of charges as one mole of $\mathrm{CaO}$, one might expect their influence on the glass network to be similar. The sequence of spectra in Figure 14 are for glasses in which the silica and alumina concentrations are held constant while the $\mathrm{Na} / \mathrm{Ca}$ ratio is varied systematically. The Raman spectra represent the vibrations of the glass-forming network so the influence of network-modifying ions appear in the spectra only indirectly. The effect of replacing $\mathrm{Na}^{+}$by $\mathrm{Ca}^{2+}$ is substantial. The high wavenumber band shifts from $919 \mathrm{~cm}^{-1}$ for the high sodium glass to $877 \mathrm{~cm}^{-1}$ for the high lime glass (Fig. 15).

\section{Interpretation}

Interpreting structure from observed Raman spectra and the interpreting durability from structure can lead to a long sequence of deductive argument. The spectra/structure correlations have a long history and an immense literature (Stebbins et al., 1995).

The prototype is silica glass, generally taken as a continuous three-dimensional network of corner-sharing silica tetrahedra. To move into the binary systems, addition of alkali or an alkaline earth to silica breaks various numbers of the $\mathrm{Si}-\mathrm{O}$-Si bridging bonds, creating non-bridging oxygens. These have a formal negative charge and so must be charged balanced by the alkali or alkaline earth, residing nearby in a high-coordination site in the network. The presence of non-bridging oxygens greatly increases the intensity of the high wavenumber tetrahedral stretching mode. Extensive investigation of binary glasses (e.g. glasses in the system $\mathrm{Na}_{2} \mathrm{O}-\mathrm{SiO}_{2}$ ) have shown that tetrahedra with one, two, or four non-bridging oxygen each have a characteristic Raman mode (Furukawa et al., 1981). The intensities of the Raman bands can be used to calculate a "speciation" diagram that shows the relative mix of tetrahedral structures as a function of alkali/silica ratio (Fig. 16-a). The wavenumbers of the individual bands varies little as a function of network depolymerization (Fig. 16-b).

Ternary glass compositions of silica, alumina and some network-modifier, particularly those in the $\mathrm{Na}_{2} \mathrm{O}-\mathrm{Al}_{2} \mathrm{O}_{3}-\mathrm{SiO}_{2}$ system, have been extensively investigated because of their relevance to the formation of igneous rocks (see, e.g., Mysen 1990, for an extensive and relatively recent review). The conventional model for aluminosilicate glass structure has the aluminum entering the network as $\mathrm{AlO}_{4}$ tetrahedra that substitute for silica tetrahedra and thus produce a continuous network which will contain $\mathrm{Si}-\mathrm{O}-\mathrm{Al}$ 


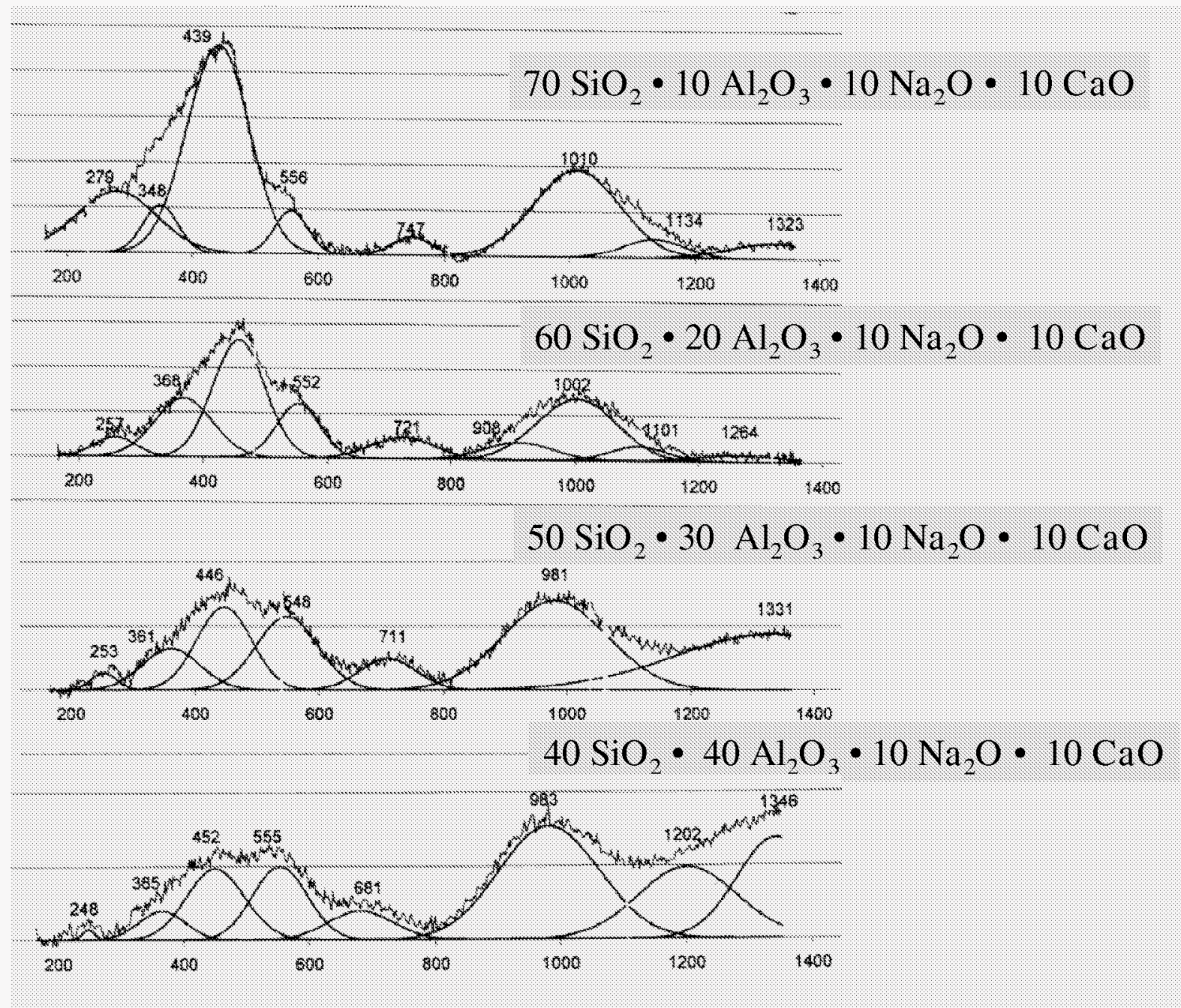

Figure 11. Raman spectra of high silica glasses with alkali plus alkaline earth fixed at 20 mole percent. 


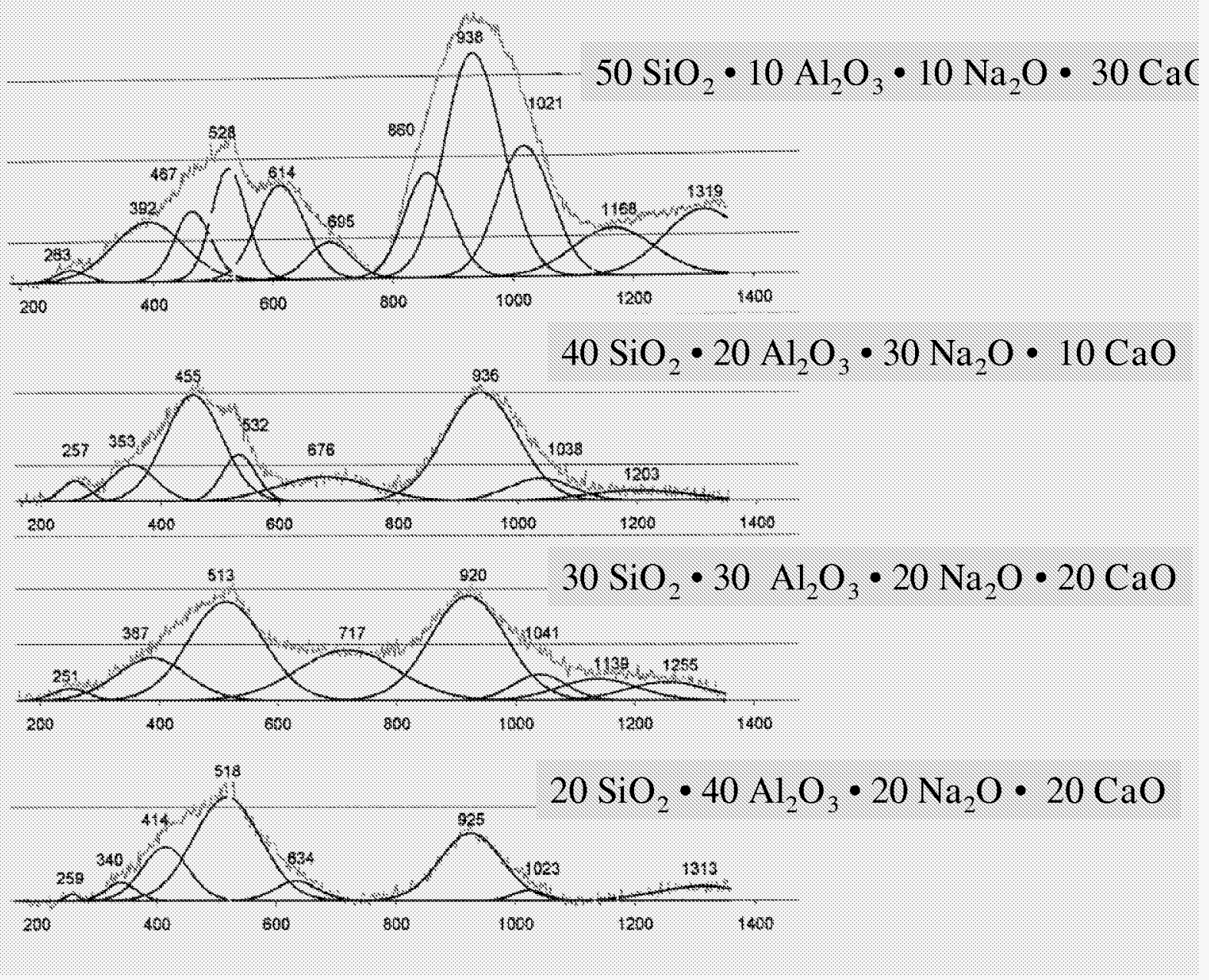

Figure 12. Raman spectra with glasses of intermediate silica concentration and alkali plus alkaline earth fixed at 40 mole percent. 


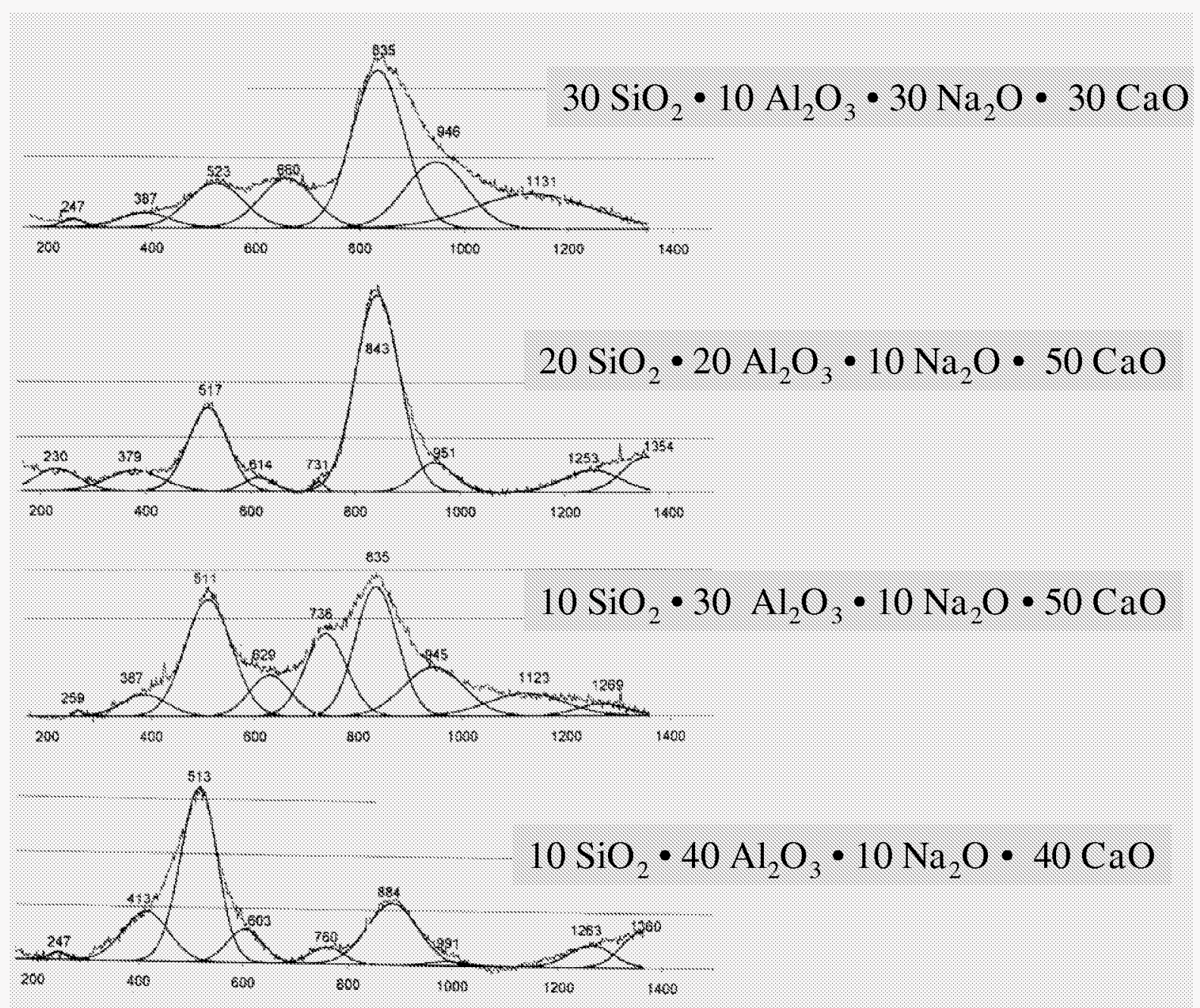

Figure 13. Raman spectra of low silica glasses with alkali plus alkaline earth fixed at 60 mile percent. 


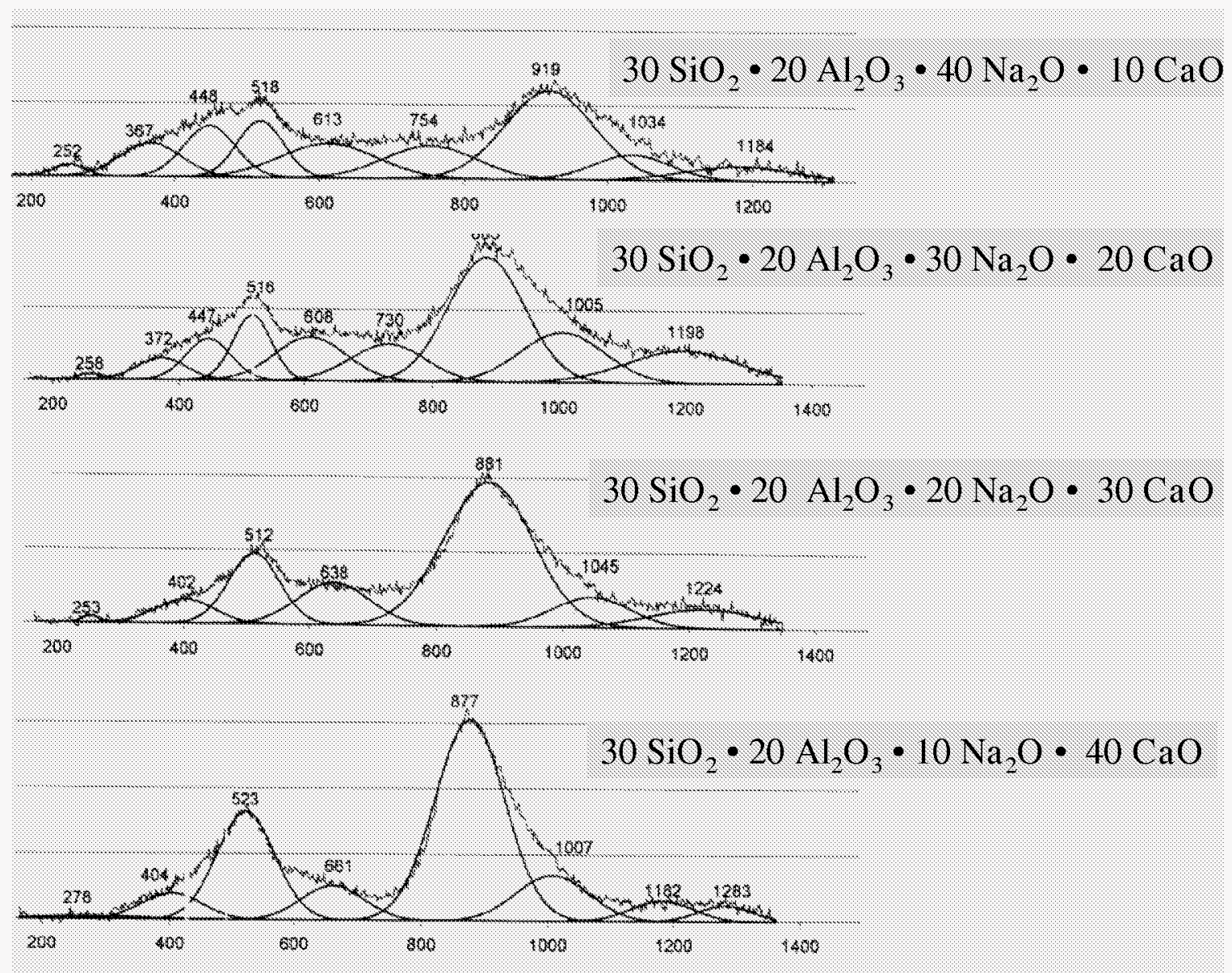

Figure 14. Thirty mole percent silica glasses with varying $\mathrm{Na}_{2} \mathrm{O} / \mathrm{CaO}$ ratio. 


\section{SOME INTERPRETATION}

\section{If THE NETWORK MODIFERS}

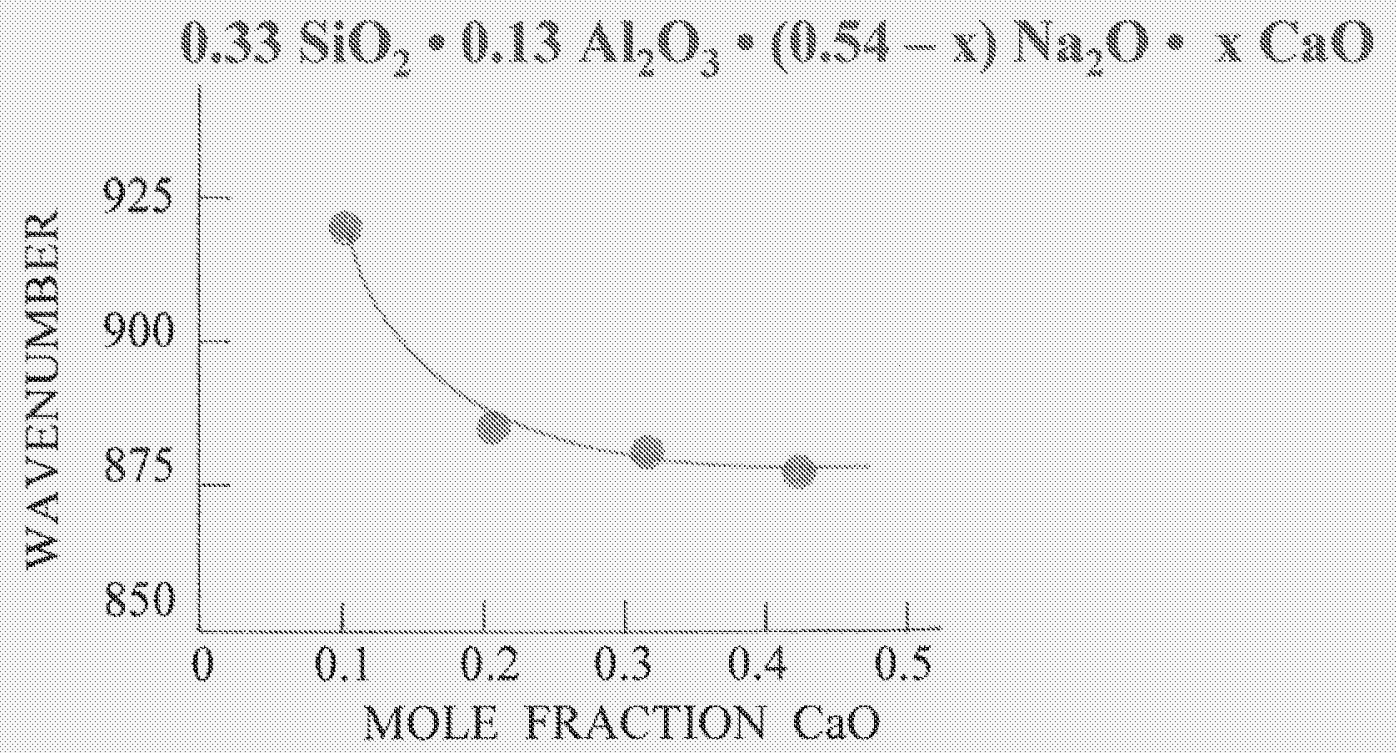

- Higher charged modifiers tend to stabilize less polymerized networks

Figure 15. Dependence of high wavenumber band on $\mathrm{CaO}$ fraction in glass with intermediate silica concentration. 
bridging bonds in addition to Si-O-Si bridging bonds. However, $\mathrm{Al}^{3+}$ is deficient by one positive charge from the $\mathrm{Si}^{4+}$ it replaces. $\mathrm{Na}^{+}$or $\mathrm{Ca}^{2+}$ must be present in sufficient quantity to provide charge balance. Alkali or alkaline earth used to provide charge balance to the alumina tetrahedra do not create non-bridging oxygens. Thus, for a fixed quantity of alkali or alkaline earth ion, addition of aluminum to the system actually increases the degree of polymerization.

The spectra of ternary alkali or alkaline earth aluminosilicate glasses differ in two important respects from the spectra of binary alkali or alkaline earth silicates. There is a tetrahedral stretching mode in the high wavenumber region and another intense band (or group of bands) in the mid-wavenumber region. Both bands are substantially broader than the corresponding bands of the binary glasses. In broad-brush terms, this implies a greater range of bond lengths and bond angles in the ternary glasses. The second difference is in the response of the high wavenumber band to the introduction of alumina into the network. Instead of creating a new band characteristic of the $\mathrm{AlO}_{4}$ tetrahedron, introduction of alumina causes the high wavenumber band to shift to lower wavenumbers as a continuous function of alumina content (Fig. 17). It appears that alumina tetrahedra and silica tetrahedra are strongly coupled and that the Raman spectrum is revealing an intermediate range structure that is larger than an isolated tetrahedron. There has been an on-going debate in the literature, reviewed briefly by White (1991), concerning the extent to which Raman bands represent extended structural units and the extent to which the observed bands are simply the sum of the contributions of individual short-range species. The species concept has proved useful for thermodynamic calculations (Halter and Mysen (2004).

Turning to the quaternary glasses that are the subject of the present investigation, we first consider the glass network. The useful parameters are the number of nonbridging oxygens per tetrahedron (NBO/T) and the alumina content. With the assumption that small, highly charged ions become part of the network and large monovalent or divalent ion act as network modifiers and occupy interstitital spaces within the network, White and Minser (1984) derived the following relationship for their investigation of natural glasses:

$$
\mathrm{NBO} / \mathrm{T}=\frac{2\left\{\left[\mathrm{Na}_{2} \mathrm{O}\right]+\left[\mathrm{K}_{2} \mathrm{O}\right]+[\mathrm{CaO}]+[\mathrm{MgO}]+[\mathrm{FeO}]-\left[\mathrm{Al}_{2} \mathrm{O}_{3}\right]-\left[\mathrm{Fe}_{2} \mathrm{O}_{3}\right]\right\}}{\left[\mathrm{SiO}_{2}\right]+2\left[\mathrm{Al}_{2} \mathrm{O}_{3}\right]+2\left[\mathrm{Fe}_{2} \mathrm{O}_{3}\right]}
$$

For the family of quaternary glasses investigated in this study the equation reduces to:

$$
\mathrm{NBO} / \mathrm{T}=\frac{2\left\{\left[\mathrm{Na}_{2} \mathrm{O}\right]+[\mathrm{CaO}]-\left[\mathrm{Al}_{2} \mathrm{O}_{3}\right]\right\}}{\left[\mathrm{SiO}_{2}\right]+2\left[\mathrm{Al}_{2} \mathrm{O}_{3}\right]}
$$

The quantities in square brackets are the number of moles of the specified component. 
These equations do not apply to the series of glasses whose spectra are shown in Figure 11. When there is insufficient alkali or alkaline earth to compensate all of the aluminum (compositions with $\left[\mathrm{Al}_{2} \mathrm{O}_{3}\right] /\left[\mathrm{Na}_{2} \mathrm{O}+\mathrm{CaO}\right]>1$ ), the structural role of aluminum must change. For the series of glasses whose spectra are show in Figures 12 and 13 , the number of non-bridging oxygens per tetrahedron varies from 0.86 to 0.0 and from 2.00 to 0.22 respectively.

\section{CONSIDERATIONS OF DURABILITY}

It has been recognized for a long time that there is at least a rough correlation between the leach rate (durability) of glasses and the number of non-bridging oxygens as calculated from glass composition (Jantzen and Plodinec, 1984). Earlier investigations of glass spectra showed that the Raman spectrum gave a measure of the number of nonbridging oxygens that correlated well with the non-bridging oxygens calculated from bulk composition using equations such as those above. This leads to a direct connection between spectroscopic behavior and glass durability (White, 1988).

The results of this study reveal several points. One is that the addition of aluminum to a silicate glass structure generally increases durability. Another is that replacing $\mathrm{Na}^{+}$by $\mathrm{Ca}^{2+}$ causes a local rearrangement of the network structure and thus is, possibly, the explanation for why soda-lime glasses are more durable than either alkali or alkaline earth binary glasses with the same number of non-bridging oxygens.

There is a final, and somewhat more subtle point. The importance of nonbridging oxygens on glass durability is that non-bridging oxygens provide a point of attack on a molecular scale for protons or water molecules. In complex glasses such as the quaternary glasses considered in this study, the glass network takes on a form that can best be described as phase-separation on a molecular scale. Aluminum tends to take primarily structural positions in which all corners of the tetrahedra form bridging bonds. In some compositions this forces the silica tetrahedra to become less polymerized. The network consists of highly polymerized regions which are resistant to chemical attack interspersed with less polymerized regions which are less durable. It is a result that warrants further investigation. 

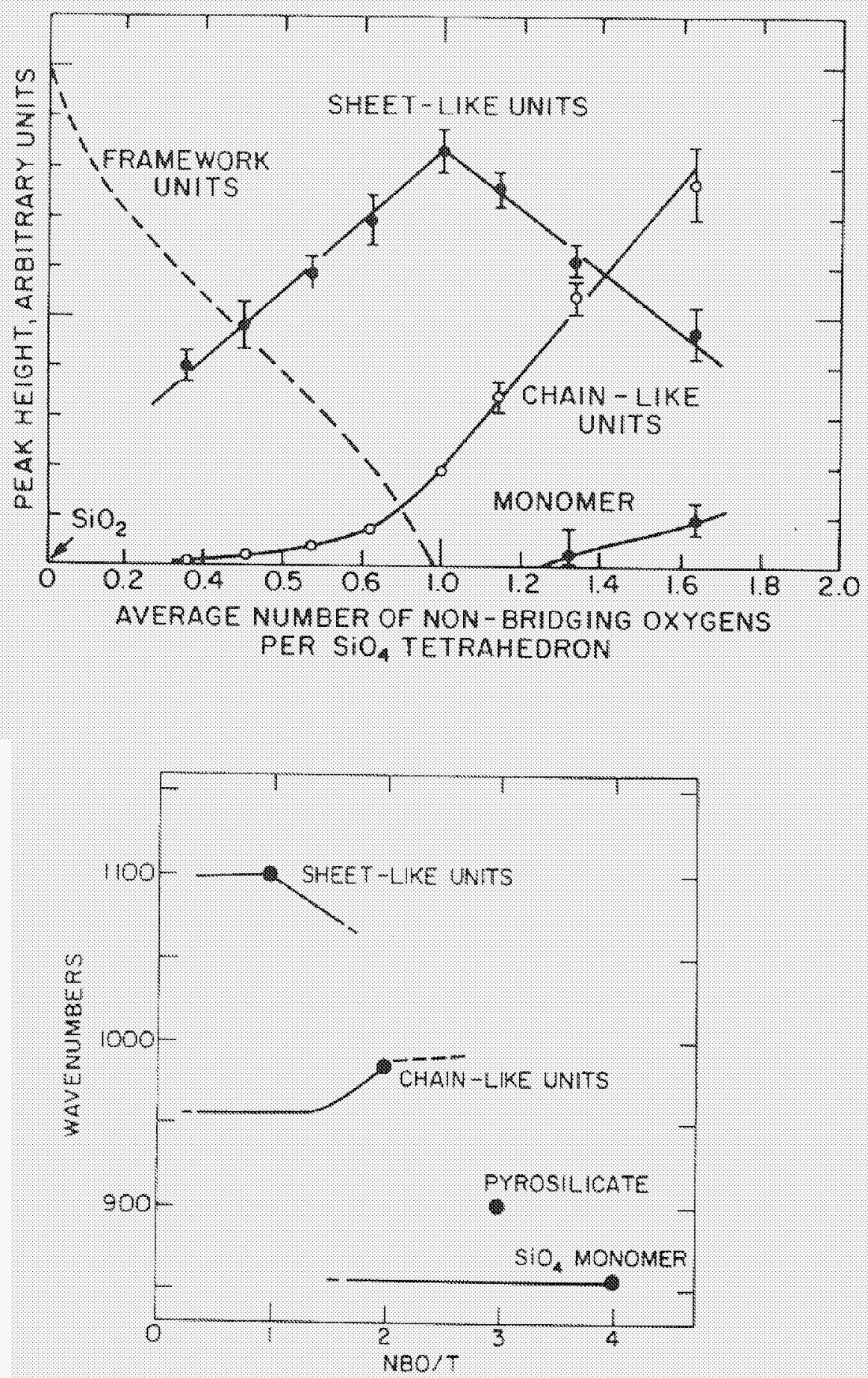

Figure 16. (A) "Speciation diagram for glasses in the $\mathrm{Na}_{2} \mathrm{O}-\mathrm{SiO}_{2}$ system. Diagram adapted from Furukawa et al. (1981). (B) Variation in band position for various $\mathrm{SiO}_{4}$ structural units as a function of the number of non-bridging oxygens. Diagram from White and Minser (1984). 


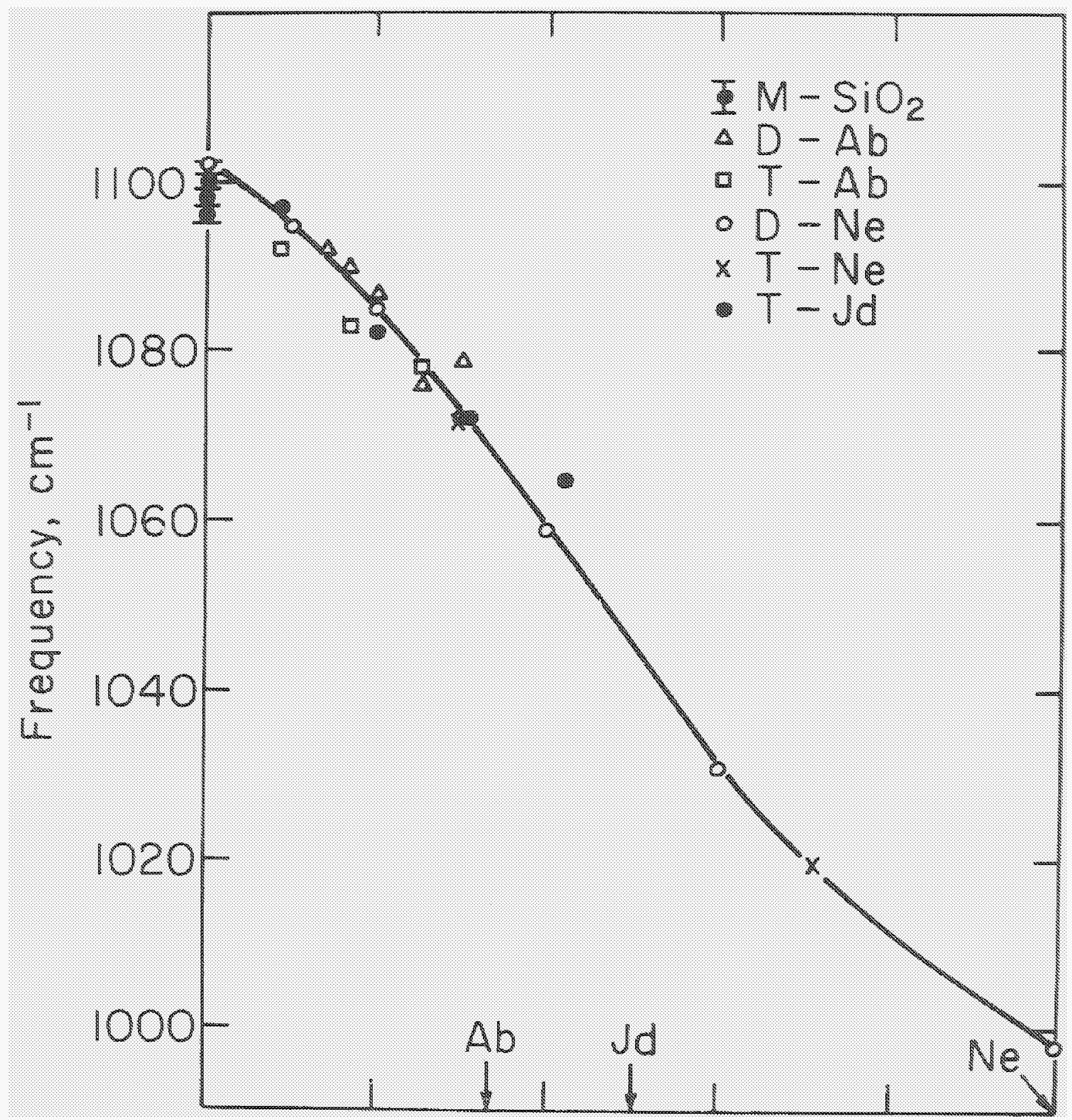

Figure 17. Position of high wavenumber band in sodium aluminosilicate glasses as a function of alumina/silica ratio. Symbols refer to corresponding crystal compositions: $\mathrm{Ab}=$ albite, $\mathrm{NaAlSi}_{3} \mathrm{O}_{8} ; \mathrm{Ne}=$ nepheline, $\mathrm{NaAlSiO}_{4} ; \mathrm{Jd}=$ jadeite, $\mathrm{NaAlSi}_{2} \mathrm{O}_{6}$. Unpublished data from this laboratory. 


\section{SYNTHESIS OF HYDROCERAMICS}

\section{A. Materials}

The first step in the synthesis of hydroceramic samples was to obtain and measure the appropriate starting materials. The necessary materials include calcined Troy clay (kaolinite), vermiculite, various chemicals and the simulated waste.

Metakaolinite derived from calcined Troy clay (kaolinite) is the major source of aluminum and silicon used for the pozzolanic reaction in the hydroceramic waste form. Since the kaolinite possesses very little or no cation exchange capacity, it must be calcined in order to render it amorphous and chemically reactive. Three-centimeter size lumps were crushed into smaller crumbs $(\sim 0.5 \mathrm{~cm})$, dried at $110^{\circ} \mathrm{C}$ in an oven overnight and then calcined for $2-3$ hours at $750^{\circ} \mathrm{C}$.

The expandable vermiculite was obtained from W. R. Grace and Co., Enoree, South Carolina, for the purpose of exchanging interlayer potassium by strontium cations. It possesses a very high ion-exchange capacity. Vermiculite flakes were ground and sieved to obtained size fractions passing through a 100 mesh $(150 \mu \mathrm{m}$ size $)$.

The chemicals used to make hydroceramics include sodium hydroxide pellets, and strontium nitrate and cesium chloride powders. The $\mathrm{NaOH}$ neutralizes the acidic capability of added water, dissolves the metakaolinite and provides the source of alkaline mineralizer that will activate metakaolinite. $\mathrm{Sr}\left(\mathrm{NO}_{3}\right)_{2}$ and $\mathrm{CsCl}$ are the major sources of surrogate strontium and cesium radionuclides. Predetermined weights of chemicals were dissolved in deionized water prior to being mixed with other solid reactant components.

The simulated waste was obtained from INEEL. The calcined wastes are of three major types: alumina calcine consisting of predominantly $90 \%$ alumina; zirconia calcine containing approximately $50 \% \mathrm{CaF}_{2}$ and $\mathrm{CaO}$ balanced mostly by alumina and zirconia; and fluorinel blend calcine originated mainly from zirconia calcine diluted with sodium nitrate and $\beta$-alumina. The simulated waste was ball-milled with zirconia balls for 24 hours to reduce particle size and increase the surface area.

\section{B. Sample Preparation}

The appropriate amount of reactants for a waste form were input into a computer program that calculates the resulting oxide ratios normalized to that of sodium oxide, solid-water ratio, hydration energies and number of non-bridging oxygens in the hydroceramic host. The main purpose of this is to adjust the components to maintain the compositions within the field of glass formation.

To make the paste, the powder components and vermiculite were blended in a Hobart mixing machine for 5-10 minutes. Sodium hydroxide solution (37.5 weight 
percent) and the solution containing $\mathrm{Sr}\left(\mathrm{NO}_{3}\right)_{2}$ and $\mathrm{CsCl}$ were combined and added to the powder. Mixing was continued until the combination had a dough-like consistency.

Small pieces of the mixture $(\sim 10 \mathrm{~g})$ were placed into $23 \mathrm{~mL}$ Teflon-lined 316 stainless steel, hydrothermal autoclave vessels. These vessels were placed into an oven for variety of times and temperatures. At the end of the reaction time, the hydrothermal vessel was removed from the oven, and allowed to air cool. The vessel was weighed both before and after it is in the oven to test for leaks.

A variety of hydroceramic samples have been made, both with and without the simulated waste. The formulations for these hydroceramic materials are shown in the Tables 2 and 3.

Table 2. Formulation for Hydroceramic (without waste)

\begin{tabular}{|l|l|}
\hline Material & Mass \\
\hline Calcined Clay & $4.33 \mathrm{~g}$ \\
\hline $\mathrm{Sr}\left(\mathrm{NO}_{3}\right)_{2}$ & $0.0225 \mathrm{~g}$ \\
\hline $\mathrm{CsCl}$ & $0.0225 \mathrm{~g}$ \\
\hline $9.375 \mathrm{M} \mathrm{NaOH}$ & $2.60 \mathrm{~g}$ \\
\hline DI water & $0.25 \mathrm{~g}$ \\
\hline
\end{tabular}

Table 3. Formulation for Hydroceramic host plus $30 \mathrm{wt} \%$ waste

\begin{tabular}{|l|l|}
\hline Material & Mass \\
\hline Calcined Clay & $5.33 \mathrm{~g}$ \\
\hline Vermiculite & $0.43 \mathrm{~g}$ \\
\hline $\mathrm{Sr}\left(\mathrm{NO}_{3}\right)_{2}$ & $0.0225 \mathrm{~g}$ \\
\hline $\mathrm{CsCl}$ & $0.0225 \mathrm{~g}$ \\
\hline $9.375 \mathrm{M} \mathrm{NaOH}$ & $2.60 \mathrm{~g}$ \\
\hline $\begin{array}{l}\text { Fluorinel blend or } \\
\text { zirconia calcine }\end{array}$ & $2.85 \mathrm{~g}$ \\
\hline DI water & $0.25 \mathrm{~g}$ \\
\hline
\end{tabular}

The hydroceramic materials that have been made differ in curing temperature and duration. The hydroceramic samples that have been prepared with incorporated waste,

Table 4. Hydroceramic Samples Types, and Time and Temperature cured

\begin{tabular}{|l|l|c|}
\hline $\begin{array}{l}\text { Waste incorporated into } \\
\text { Hydroceramic Sample }\end{array}$ & Temperature & Duration \\
\hline \multirow{2}{*}{ No Waste } & $125^{\circ} \mathrm{C}$ & $2,4,6,12,18,24,36$ and $48 \mathrm{hrs}$ \\
\cline { 2 - 3 } & $150^{\circ} \mathrm{C}$ & $2,4,6,12,18,24,36$ and $48 \mathrm{hrs}$ \\
\hline
\end{tabular}




\begin{tabular}{|l|l|l|}
\hline (Hydroceramic host only) & $175^{\circ} \mathrm{C}$ & $2,4,6,12,18,24,36$ and $48 \mathrm{hrs}$ \\
\cline { 2 - 3 } & $200^{\circ} \mathrm{C}$ & 24,30 and $48 \mathrm{hrs}$ \\
\hline \multirow{2}{*}{ Run 64 waste } & $75^{\circ} \mathrm{C}$ & 48 and $60 \mathrm{hrs}$ \\
\cline { 2 - 3 } & $125^{\circ} \mathrm{C}$ & 12 and $24 \mathrm{hrs}$ \\
\hline Run 74 waste & $75^{\circ} \mathrm{C}$ & $24,36,48$ and $60 \mathrm{hrs}$. \\
\cline { 2 - 3 } & $125^{\circ} \mathrm{C}$ & 12 and $24 \mathrm{hrs}$ \\
\hline
\end{tabular}

incorporate one of two different types of waste, entitled run 64 and run 74. Table 4 describes the samples that have been made, including the type of waste incorporated (if any), the time, and the temperature at which each sample has been cured. The end products were cubes, about $1.5 \mathrm{~cm}$ on an edge.

C. Conversion of Hydroceramic into Glass

The fusability of the hydroceramic samples was tested by holding an oxyhydrogen torch against a corner of the cubes. The cementious material melted into a dark green glass. As the glass lost viscosity during melting, a bead of glass formed dissociated from the bulk of the sample and formed into a spherical bead as it cooled into glass. The hydroceramic samples melted quite easily with the torch (far easier than the glass samples that have been made). These beads are suitable for spectroscopic characterization and for comparison with hot isostatic pressed material.

\section{WASTE FORM DURABILITY AND LEACH TESTING}

As sample preparation is underway, work has been started on developing methods for testing the durability of hydroceramics, glasses, and glass ceramics. These tests will be based on the MCC series of test procedures developed for nuclear waste glasses. Test protocols have been reviewed and are described briefly below.

\section{A. MCC-1 Static Leach Test}

The MCC-1 Static Leach Test is intended to test chemical durability of simulated and radioactive monolithic, inorganic waste forms (glass, ceramic, etc). The tests are performed at temperatures not exceeding $100^{\circ} \mathrm{C}$ under low SA/V (surface area/volume) conditions. Normalized elemental mass loss is calculated based on results of exposure to specific aqueous solutions (leachants). Reference leachants are high purity water, silicate/bicarbonate solutions and brine solutions, the latter of which provide a geochemical environment similar to those anticipated for geologic repositories. Specimens to be placed in reference leachants are of known volume and surface area, are exposed for defined temperatures and times, and are not agitated.

B. MCC-3 Agitated Powder Leach Test

The MCC-3 Agitated Powder Leach Test is also designed to evaluate chemical durability of nuclear waste forms. However, the specimens are powdered samples 
derived from the monolithic, inorganic waste forms, resulting in a higher SA/V ratio. The same reference leachants are used, with potential for use of additional leachants such as site-specific expected ground waters or other fluids. The Agitated Powder Leach Test Method differs from The Static Leach Test Method in that in the former, the tests to determine maximum chemical concentrations of extracted ions are performed under steady state conditions in closed, agitated containers, and at temperatures varying between $40^{\circ} \mathrm{C}$ and $190^{\circ} \mathrm{C}$. More rapid reaction kinetics are expected in performing MCC-3 test due to the increased SA/V ratio.

\section{REFERENCES}

Furukawa, T., K.E. Fox and W.B. White (1981) Raman spectroscopic investigation of the structure of silicate glasses. III. Raman intensities and structural units in sodium silicate glasses. Journal of Chemical Physics 75, 3226-3237.

Gougar, M.L.D., D.D. Siemer and B.E. Scheetz (1995) Vitrifiable concrete for disposal of spent nuclear fuel reprocessing waste at I.N.E.L. Scientific Basis for Nuclear Waste Management XIX, Mater. Res. Soc. Symposium Proceedings.

Halter, W.E. and B.O. Mysen (2004) Melt speciation in the system $\mathrm{Na}_{2} \mathrm{O}-\mathrm{SiO}_{2}$. Chemical Geology 213, 115-123.

Jantzen, C.M. and M.J. Plodinec (1984) Thermodynamic model of natural, medieval, and nuclear waste glass durability. Journal of Non-Crystalline Solids 67, 207-223.

Mysen, B.O., L.W. Finger, D. Virgo, and F.A. Siefert (1982) Curve-fitting of Raman spectra of silicate glasses. American Mineralogist 67, 686-695.

Mysen, B.O. (1990) Relationship between silicate melt structure and petrologic processes. Earth-Science Reviews 27, 281-365.

Stebbins, J.F., P.F. McMillan and D.B. Dingwell (Eds.) Structure, Dynamics and Properties of Silicate Melts. Reviews in Mineralogy, Vol. 32, 616 pp.

White, W.B. and D.G. Minser (1984) Raman spectra and structure of natural glasses. Journal of Non-Crystalline Solids 67, 45-59.

White, W.B. (1988) Glass structure and glass durability. Materials Research Society Symposium Proceedings 125, 109-114.

White, W.B. (1991) Spectroscopic determination of nanoscale structures in glasses and gels. In Glasses for Electronic Applications, K.M. Nair, Ed., American Ceramic Society Transactions 20, 1-17. 